\title{
Probing Interactions in Complex Molecular Systems through Ordered Assembly
}

J. J. De Yoreo, M. C. Bartelt, C. A. Orme, A. Villacampa, B. L. Weeks, A. E. Miller

January 31, 2002

U.S. Department of Energy

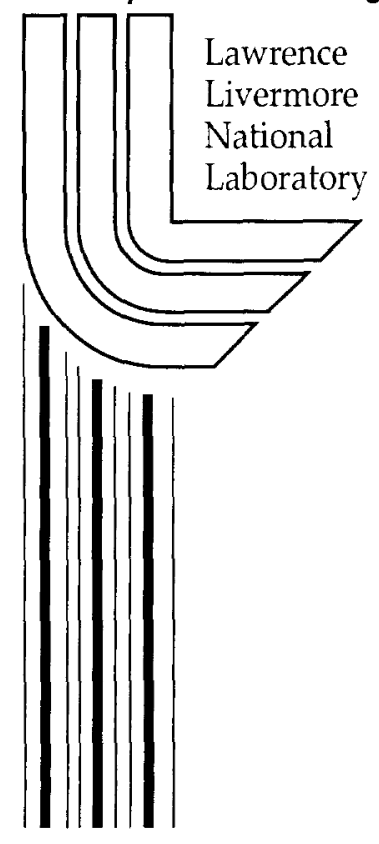




\section{DISCLAIMER}

This document was prepared as an account of work sponsored by an agency of the United States Government. Neither the United States Government nor the University of California nor any of their employees, makes any warranty, express or implied, or assumes any legal liability or responsibility for the accuracy, completeness, or usefulness of any information, apparatus, product, or process disclosed, or represents that its use would not infringe privately owned rights. Reference herein to any specific commercial product, process, or service by trade name, trademark, manufacturer, or otherwise, does not necessarily constitute or imply its endorsement, recommendation, or favoring by the United States Government or the University of California. The views and opinions of authors expressed herein do not necessarily state or reflect those of the United States Government or the University of California, and shall not be used for advertising or product endorsement purposes.

This work was performed under the auspices of the U. S. Department of Energy by the University of California, Lawrence Livermore National Laboratory under Contract No. W-7405-Eng-48.

This report has been reproduced directly from the best available copy.

Available electronically at http://www.doc.gov/bridge

Available for a processing fee to U.S. Department of Energy

And its contractors in paper from

U.S. Department of Energy

Office of Scientific and Technical Information

P.O. Box 62

Oak Ridge, TN 37831-0062

Telephone: (865) 576-8401

Facsimile: (865) 576-5728

E-mail: reports@adonis.osti.gov

Available for the sale to the public from

U.S. Department of Commerce

National Technical Information Service 5285 Port Royal Road Springfield, VA 22161

Telephone: (800) 553-6847

Facsimile: (703) 605-6900

E-mail: orders@ntis.fedworld.gov

Online ordering: http://www.ntis.gov/ordering.htm

OR

Lawrence Livermore National Laboratory

Technical Information Department's Digital Library

http://www.llnl.gov/tid/Library.html 


\section{Probing Interactions in Complex Molecular Systems through Ordered Assembly}

01-ERD-040

J. J. De Yoreo, M. C. Bartelt, C.A. Orme, A. Villacampa, B.L. Weeks, A.E. Miller

\section{Background and purpose}

Emerging from the machinery of epitaxial science and chemical synthesis, is a growing emphasis on development of self-organized systems of complex molecular species. The nature of self-organization in these systems spans the continuum from simple crystallization of large molecules such as dendrimers and proteins, to assembly into large organized networks of nanometer-scale structures such as quantum dots or nanoparticles. In truth, self-organization in complex molecular systems has always been a central feature of many scientific disciplines including fields as diverse as structural biology, polymer science and geochemistry. But over the past decade, changes in those fields have often been marked by the degree to which researchers are using molecularscale approaches to understand the hierarchy of structures and processes driven by this ordered assembly. At the same time, physical scientists have begun to use their knowledge of simple atomic and molecular systems to fabricate synthetic self-organized systems. This increasing activity in the field of self-organization is testament to the success of the physical and chemical sciences in building a detailed understanding of crystallization and epitaxy in simple atomic and molecular systems, one that is soundly rooted in thermodynamics and chemical kinetics. One of the fundamental challenges of chemistry and materials science in the coming decades is to develop a similarly wellfounded physical understanding of assembly processes in complex molecular systems.

Over the past five years, we have successfully used in situ atomic force microscopy (AFM) to investigate the physical controls on single crystal epitaxy from solutions for a wide range of molecular species. More recently, we have combined this method with grazing incidence X-ray diffraction and kinetic Monte Carlo modeling in order to relate morphology to surface atomic structure and processes. The purpose of this proposal was to extend this approach to assemblies of three classes of "super molecular" nanostructured materials. These included 1) dendrimers, 2) DNA bonded nano-particles, 


\section{1-ERD-040 \\ mat'ls synthesis and characterization}

and 3) colloids, all of which form solution-based self-organizing systems. To this end, our goals were, first, to learn how to modify models of epitaxy in small molecule systems so that they are useful, efficient, and applicable to assembly of super-molecular species; and, second, to learn how systematic variations in the structure and bonding of the building blocks affect the surface kinetics and energetics that control the assembly process and the subsequent dynamic behavior of the assembled structures. AFM imaging provided experimental data on morphology and kinetics, while kinetic Monte Carlo (KMC) simulations related these data to molecular-scale processes and features.

\section{Methods}

In situ AFM measurements were performed using an experimental arrangement like that shown schematically in Fig. 1. Solution of known composition and temperature was flowed through a cell within the AFM containing the crystal to be imaged. Image sizes were chosen to optimize our ability to analyze step speeds and deviations of the step edges from straight lines. Flow rates were maintained at sufficiently high levels to ensure that step advancement was not limited by mass transport in the bulk fluid. Two types of data were collected. In the first, the AFM tip was scanned rapidly along an axis roughly perpendicular to the step and slowly along the orthogonal direction to give a real space image of the step. Sequential images enabled us to extract step speeds and spatial fluctuations in step shape and interstep spacing (terrace width). In the second type of measurement, the slow-scan axis was disabled so that the resulting image was a time profile of a point along the step. From this time profile, temporal fluctuations in the step position or, equivalently, fluctuations in step speed could be determined. Examples of the two types of measurements are shown in Fig. 2.

KMC simulations implement models of molecular attachment to or detachment from growing or dissolving surfaces during solution flow. In order to be predictable, these models must include as many relevant (potentially rate-limiting) processes as feasible. A minimum set of such processes, and a reasonable distinction in their rates, for individual molecules approaching a step are shown schematically in Fig.3. For anisotropic systems, an independent set of rates must be incorporated for each different type of step. Quantitative differences found between the steps reflect differences in their 
chemical structure. Several of those rates are actually simply related by the strength of the bonds that a molecule makes with other neighboring molecules along $\left(\mathrm{E}_{\|}\right)$or perpendicular $\left(E_{\perp}\right)$ to the step (see the inset in Fig.3). Other rates (e.g., for attachment) contain information about interactions with the solvent. These features dramatically simplify the models by reducing the number of initially unknown parameters that need to be adjusted in the simulations. In practice, the rates are estimated by matching the simulation results with experiment for all the measured quantities (step speeds, roughness, stiffness, etc.).

Methods for pre-patterning of the substrate prior to crystallization are motivated by Because of the need to create a nucleus nuclei when trying to induce ordered assembly of hard-to-crystallize species. Thus, we investigated the use of chemical patterns at surfaces as templates, first for nucleation of colloidal structures. We used the force microscope to create these patterns via a method known as dip-pen nanolithography (DPN). To perform DPN, an AFM tip is "inked" with a chemical of interest and brought into contact with a surface. The ink molecules flow from the tip onto the surface analogous to a fountain pen. This method is capable of producing nanostructures down to $15 \mathrm{~nm}$ in diameter and separated by as little as $5 \mathrm{~nm}$. While, in principle, nearly any "ink" or "paper" can be used, as has been demonstrated with dendrimers, conducting polymers, organic dyes, antibodies, and gold on silicon, by far the most common application of DPN is the patterning of thiol "inks" on gold "paper". An example of a dots of controlled sizes and separations formed by this method is shown in Fig. 4a.

\section{Results}

Our activities focused on three areas: $\mathrm{KMC}$ simulations to elucidate the role of anisotropy and intermolecular bond strength on step morphology and kinetics, analyses of step fluctuations recorded in AFM images to extract the basic physics of mass transport at step edges, and the use of AFM to make chemical patterns as templates for initiating ordered assembly of colloids. All of these areas yielded significant results.

KMC simulations: 
mat'ls synthesis and characterization

$\mathrm{KMC}$ simulations show conclusively that it is possible to relate (even small) variations in step speed and roughness to variations in the strength and anisotropy of intermolecular bonds at the step. Figure 5a illustrates this observation with simulation results for three combinations of bond strengths parallel $\left(E_{\|}\right)$and perpendicular $\left(E_{\perp}\right)$ to the step. For fixed $E_{\|}$or $E_{1}$, the step is smoother and slower the smaller the value of $E_{\|}$or $E_{\perp}$, respectively. This result is easy to understand: weak bonds facilitate detachment from the step, a process which creates more kinks that are unstable against detachment. Therefore, the step is slowed down and cannot maintain a large population of kink sites (the main source of roughness). Bond anisotropy plays an important role: no matter how strong $\mathrm{E}_{\|}$ may be, a weak $E_{\perp}$ limits the population of new growth sites, the isolated molecules attached to the step by just a perpendicular bond. To first, good approximation, this population uniquely determines the step speed and roughness. The main role of $E_{\|}$is to stabilize connected clusters of these new growth sites along the step, whose net effect is to speed up the step (see Fig.5a).

The KMC simulations also show that when the bond strengths are much higher than the thermal energy $k T$, roughening at the step edges leads to nonlinear dependences of step speed on solute concentration. Fig. 5b shows the non-linear nature of the dependence of both the step roughness and step speed on concentration for various values of bond strength perpendicular to the step. In contrast, in the limit of bond strength that is small compared to $\mathrm{kT}$, the dependence reaches the limit of roughness and the concentration dependence of the step speed becomes linear.

\section{AFM measurements:}

Our in situ AFM images are extremely rich in information about the relationship between morphology and binding anisotropies and surface (step) dynamics. To illustrate our ability to quantify this relationship, we imaged the growth of a brushite (calcium phosphate dihydrate) crystal. On the (010) surface of this crystal three distinct step directions are expressed, as shown in the schematic of Fig. 6a. Figure $6 \mathrm{~b}$ shows a corresponding AFM image of this surface. From this image one can clearly see the differences in step roughness. Step speeds for these three step directions (not shown) vary 


\section{1-ERD-040 \\ mat'ls synthesis and characterization}

by a factor of seven from the slowest to the fastest (the fastest being also the roughest). Both the terrace width distributions and step edge fluctuations also show significant differences. One way to analyze these fluctuations is to measure correlations in the height of the step relative to its average position, at different positions along the step (for fixed time) and at different times for a fixed location on the step. While the former is strictly impossible, due to the finite time to scan an image with the AFM tip, it is feasible for steps with relatively slow dynamics.

From these data, information about step edge energetics (in particular the cost of bending or creating kinks at the step) and mass transfer can be extracted. Spatial and temporal height-height correlation functions, $G(x)$ and $G(t)$, respectively, for one of the step directions of brushite are shown in Fig. 7. The linear dependence of $G(x)$ is expected for fluctuations controlled by a simple one-dimensional random walk of open sites along the step. The exponent of 0.5 found for $G(t)$ is expected when the transfer of molecules from one portion of the step to another is controlled by the rate of diffusion of those molecules along the terrace or through the solution, diffusion along the step being more difficult.

\section{Organization of colloids:}

Using COOH-terminated alkane thiols as the ink, we made a series of DPN patterns. We then attempted to control the subsequent deposition of nanoscale adsorbates by using $40 \mathrm{~nm}$ amine-terminated polystyrene spheres as the colloidal species. After a significant amount of experimentation with solution chemistry and post-depositional washing, we were able to controllably produce patterning of deposited beads. Figure 8 shows an example in which the thiol dots were widely spaced and larger than the beads. Consequently, each dot holds more than one bead. While we did not advance to the point where we could create close-packed arrays of colloids before the end of the project, this result is proof that this approach can succeed.

\section{Conclusion}

This project resulted in the development and testing of new methods for investigating the controls on ordered assembly in complex molecular systems. We 
envision application to a number of LLNL programmatic activites. The KMC methods developed here have direct application to a significant problem in ICF laser systems, that of laser-induced damage in $\mathrm{KH}_{2} \mathrm{PO}_{4}$ crystals caused by the incorporation of nanometerscale particles, as well as the differential incorporation of ionic complexes that modify the refractive and absorptive properties of the crystals. The AFM results have a direct bearing on the growth of energetic crystals for high explosives. These highly anisotropic molecular crystals exhibit a destructive phase transition that appears to nucleate at defects. The AFM results shown here demonstrate that the type and distribution of defects in these crystals, as well as their mechanism of creation, can be understood with systematic AFM analyses. Finally, ability to assemble nanometer-scale particles at surfaces has far-reaching consequences in both the areas of proteomics and counterterrorism. Assembly of macromolecules into ordered arrays may allow one to avoid the problem of bulk crystallization, which is currently the rate-limiting step in protein structure determination. Furthermore, developing methods of controlling attachment of specific viruses, proteins, or spores can lead to highly-compact and sensitive means of bio-agent detection.

\section{Figure Captions}

Fig. 1 - Schematic of experimental set-up showing (a) flow and (b) temperature control systems for AFM experiments.

Fig. 2 - AFM images of atomic steps on a brushite crystal collected while scanning along (a) both axes and (b) just the horizontal axis. While (a) shows the real shape of the step, in (b) the vertical axis represents time.

Fig. 3 - Schematic of the simulation model for attachment and detachment of molecular species at steps of molecular crystals.

Fig. 4 - Pattern of COOH-terminated alkane thiol dots on gold formed by DPN showing the dependence of dot size on contact time. 
Fig. 5 - (a) Result of kinetic Monte Carlo simulation indicating the dependence of step roughness and propagation speed on bond strengths $E_{\|}$and $E_{\perp}$, parallel and perpendicular, respectively, to an initial step (dotted line at left); the three jagged lines show the position of the step, at equal times, for the various bond strengths shown. Units of position $x$ and $y$ are number of lattice sites. (b) dependence of step speed and roughness on concentration for various values of $E_{\perp}$.

Fig. 6 - (a) Atomic structure of brushite surface where the lines of truncation of the surface indicate the directions of the steps observed in (b). (b) AFM image of growth source on brushite surface showing correspondence with step directions indicated in (a).

Fig. 7 - (a) Spatial and (b) temporal height-height correlation functions for a step on the brushite surface, showing the linear dependence of $G(x)=[y(x)-y(0)]^{2}$ and the power law dependence of $G(t)=[y(t)-y(0)]^{2} \cdot y(x)$ or $y(t)$ is the position of the step relative to a reference value $\mathrm{y}(0)$.

Fig. 8 - Pattern of amine terminated polystyrene beads attached to $\mathrm{COOH}$-terminated alkane thiol dots on gold. 

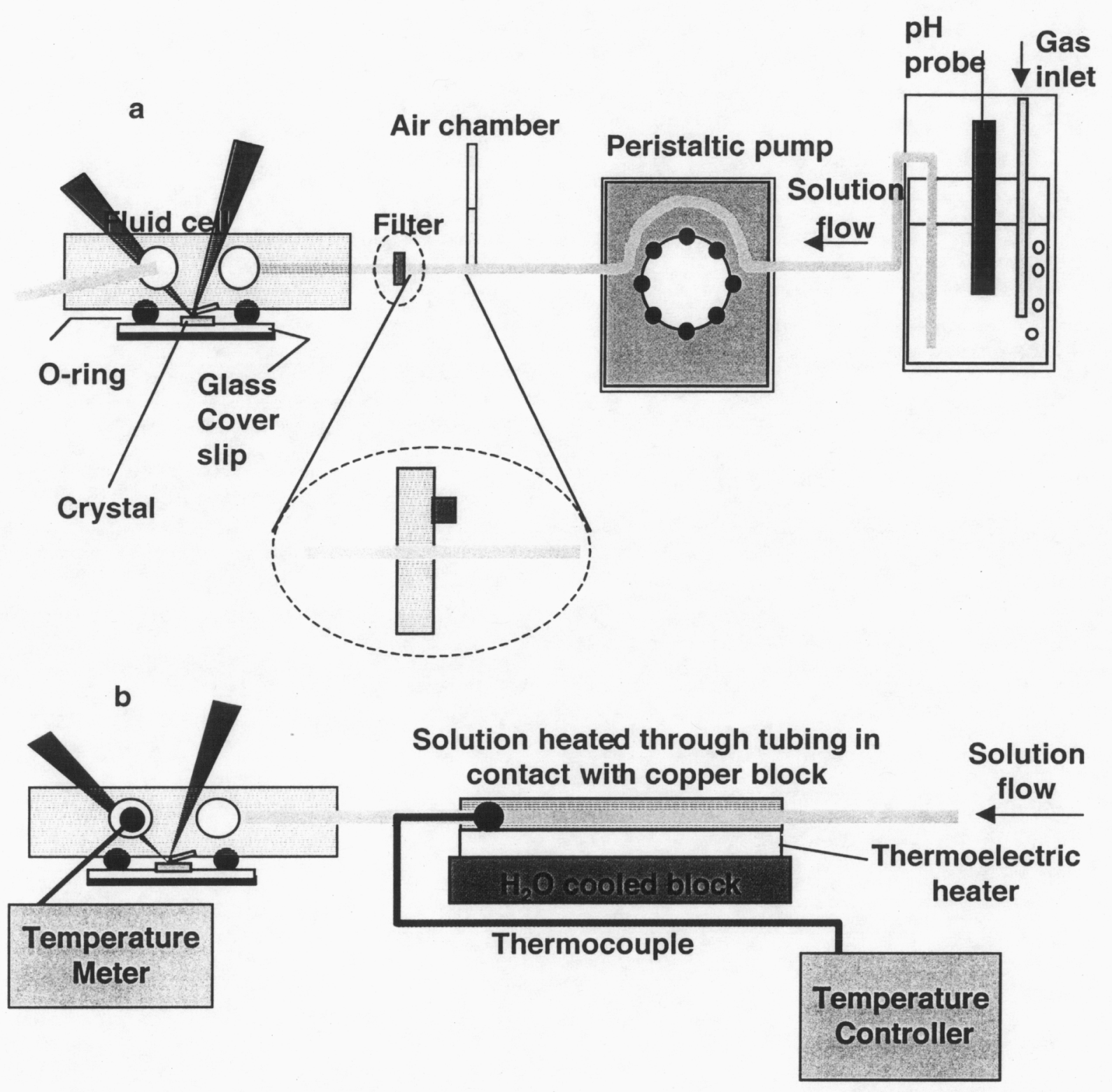


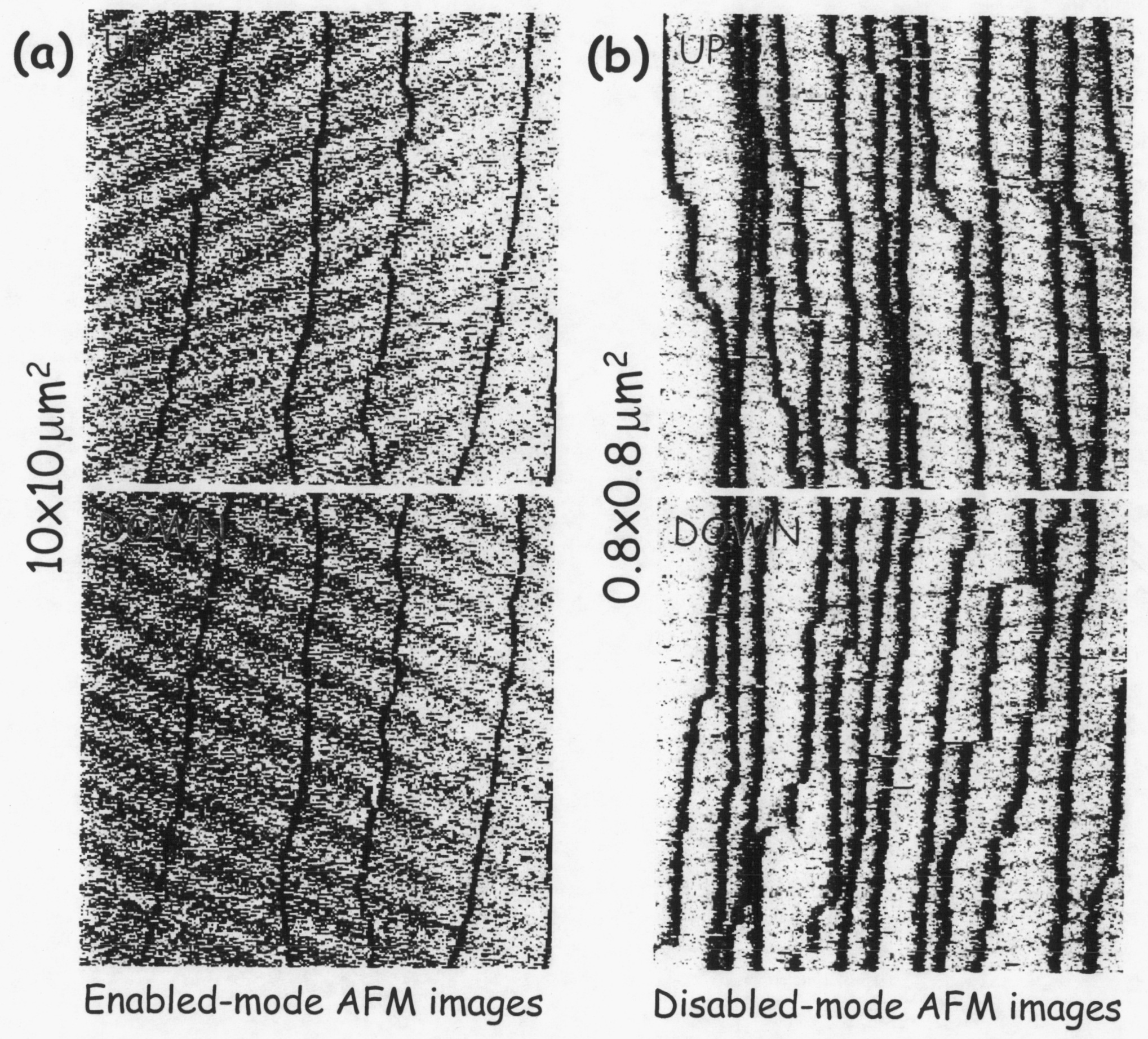




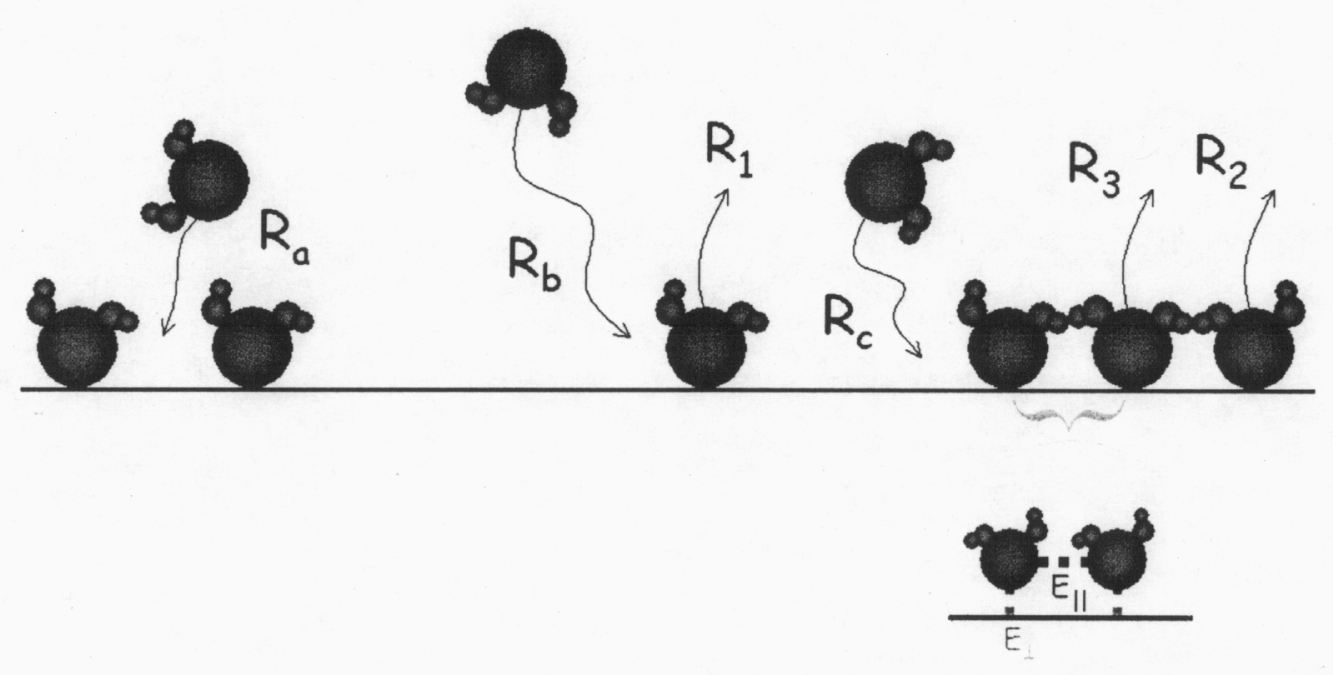

LDRD Final Report: Fig.3 


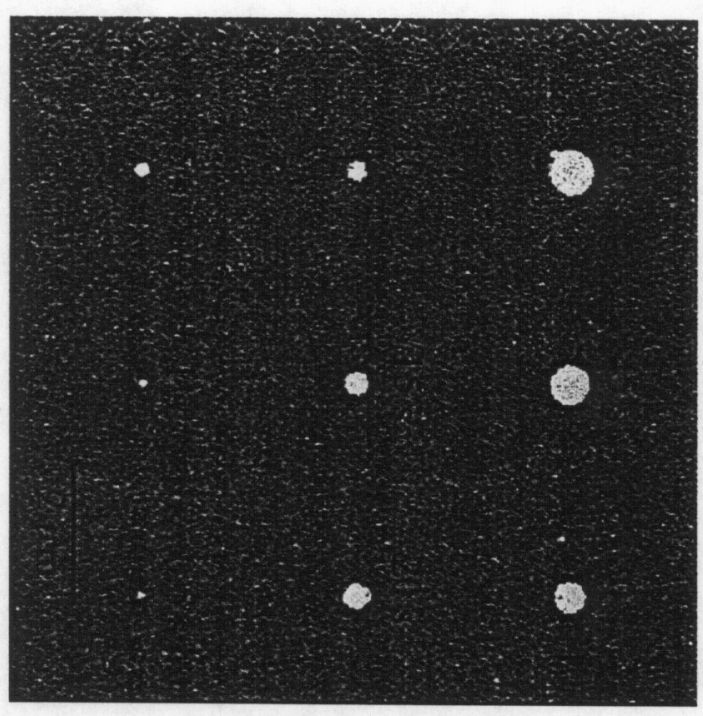




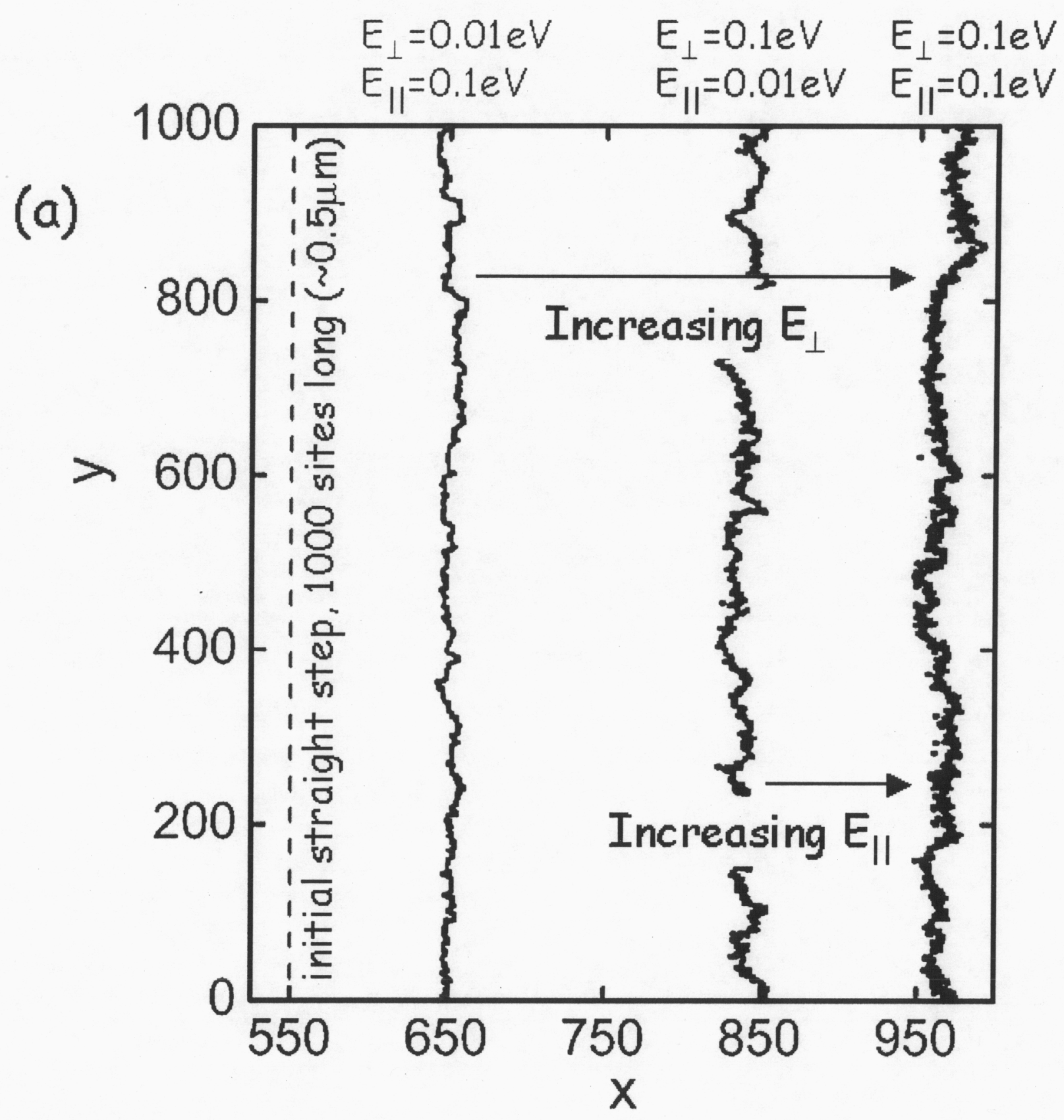


(120)
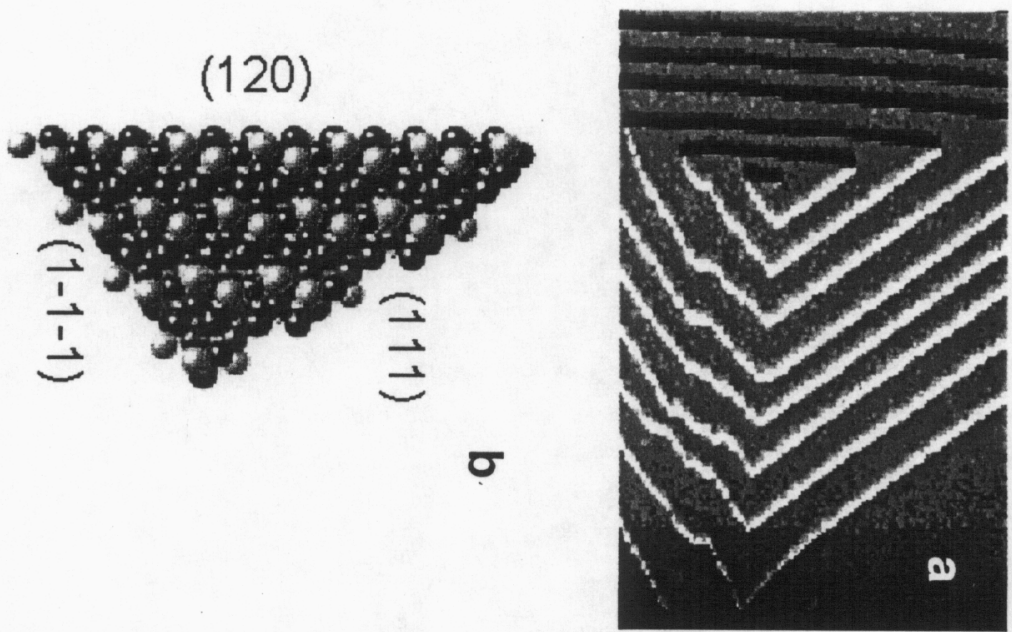

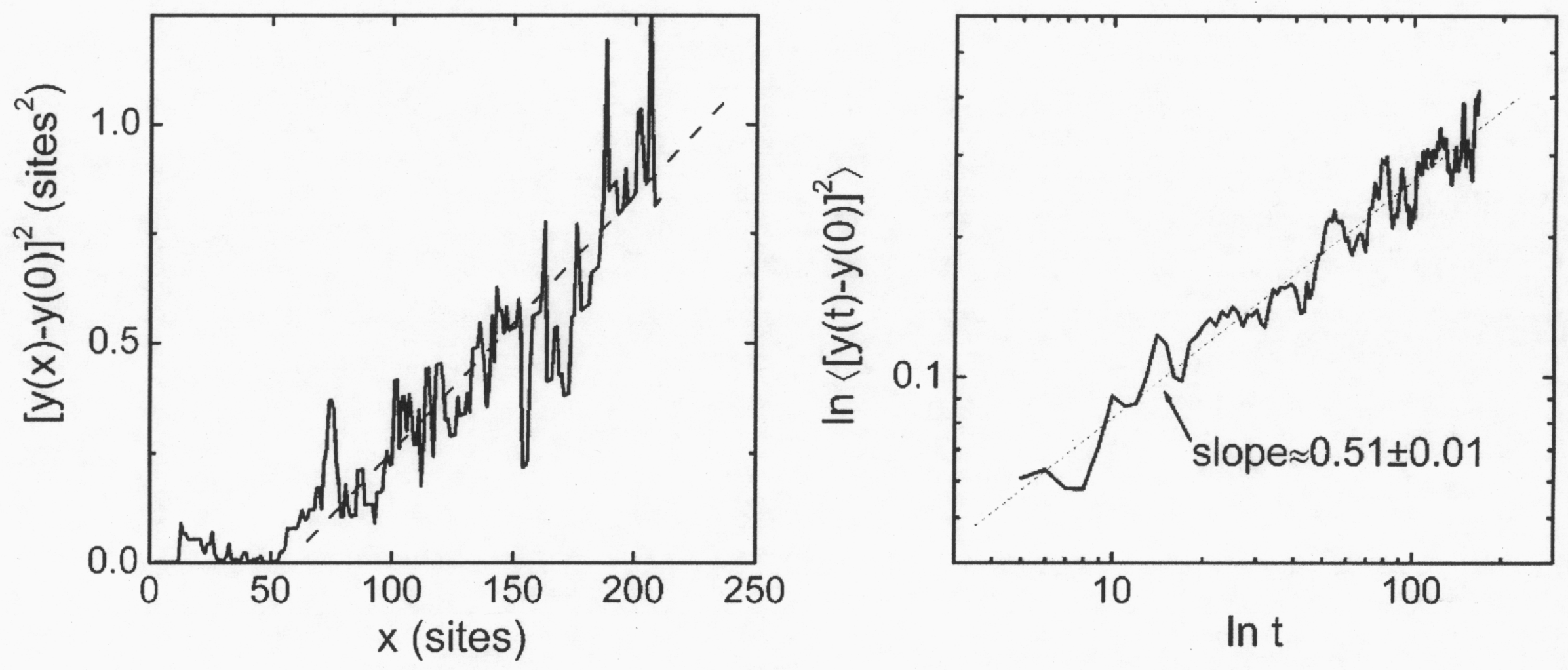


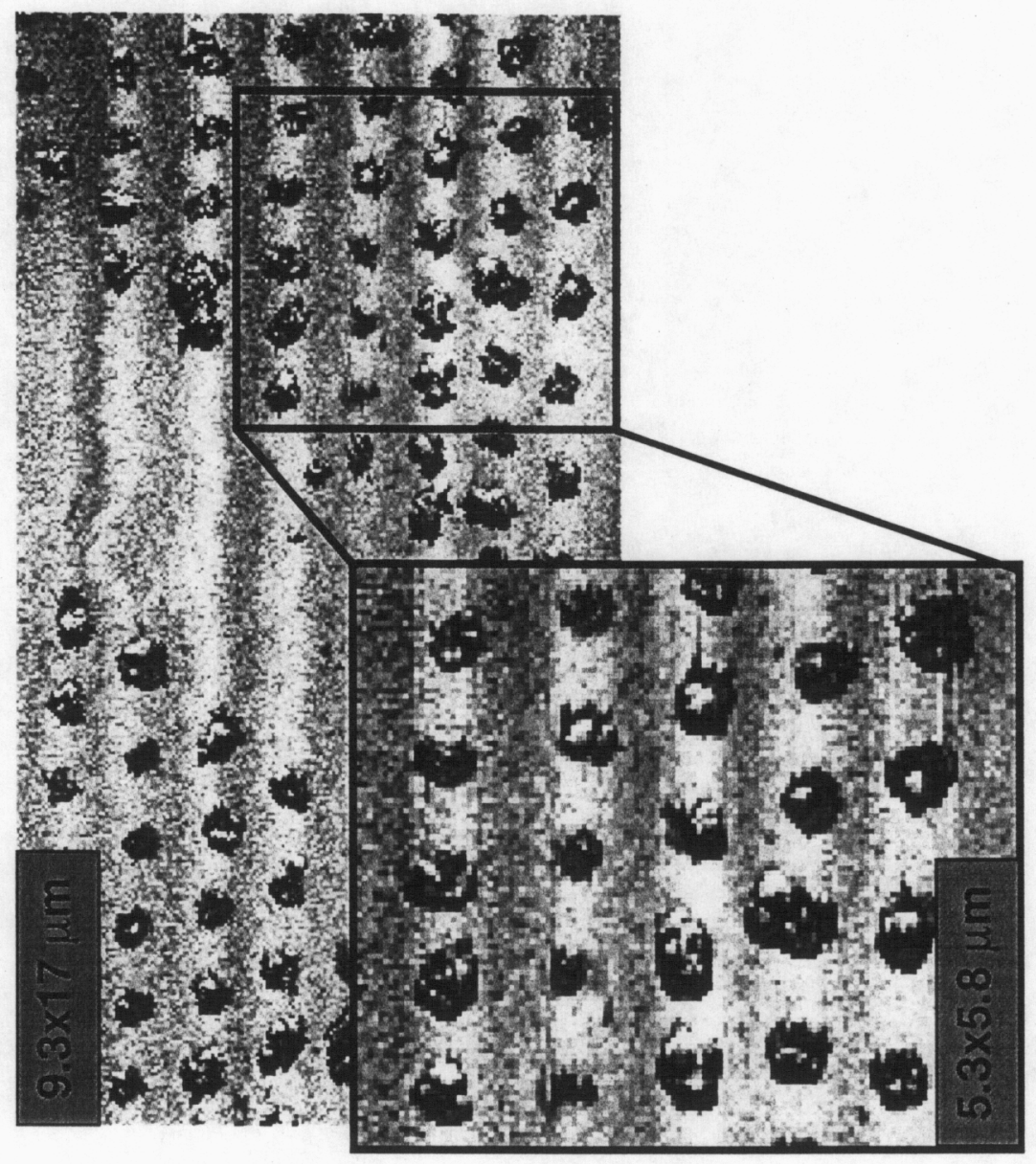

